\title{
Semánticas de la transformación: Desafíos y potencialidades de la salud mental en la provincia de San Luis, Argentina
}

Semânticas da transformação: desafios e potencialidades da saúde mental na província de San Luis, Argentina

Semantics of transformation: challenges and potentialities of mental health in the province of San Luis, Argentina

\section{Romina Prat}

Licenciada en Psicología

Hospital de Salud Mental y Adicciones de la Provincia de San Luis, Argentina.

Universidad Nacional de Cordoba, Argentina.

Márcio Mariath Belloc

Doutorado em Antropologia

Universidade Federal do Pará

\section{Resumen}

El presente artículo trata de una reflexión sobre las bases de una investigación y un análisis de sus resultados preliminares. La investigación se enmarca en la Región Sanitaria I de la provincia de San Luis, Argentina, y trata sobre los desafíos y potencialidades da la implementación de la red de atención en el ámbito de la salud mental. Es un estudio descriptivo en tanto intenta caracterizar y describir la situación actual de los servicios de salud de una región sanitaria teniendo en cuenta las necesidades y recursos de cada servicio con el objetivo de lograr una aproximación a la situación actual de los servicios de salud de la región. Una realidad que demanda transformaciones semánticas para alcanzar respuestas clínicas y sociales más inclusivas y participativas en relación a la salud mental.

Palabras clave: Salud Pública; Salud Mental; Sistemas de salud; Apoyo social.

\section{Resumo}

O presente artigo trata de uma reflexão sobre as bases de una investigação e uma análise de seus resultados preliminares. A investigação está demarcada na Região Sanitária I da província de San Luis, Argentina, e trata sobre os desafios e potencialidades da implementação da rede de atenção no âmbito da saúde mental. É o estudo descritivo em tanto intenta caracterizar e descrever a situação atual dos serviços de saúde de uma região sanitária, levando em consideração as necessidades e recursos de cada serviço com o objetivo de lograr uma aproximação à situação atual da rede de saúde da região. Uma realidade que demanda transformações semânticas para alcançar respostas clínicas y sociais mais inclusivas e participativas em relação à saúde mental. 
Palavras-chave: Saúde Pública; Saúde Mental; Sistemas de Saúde; Apoio Social.

\section{Abstract}

This article deals with a reflection on the bases of an investigation and an analysis of its preliminary results. The investigation is demarcated in the Sanitary Region I of the province of San Luis, Argentina, and deals with the challenges and potential of implementing the care network in the context of mental health. It is the descriptive study that attempts to characterize and describe the current situation of health services in a health region, taking into account the needs and resources of each service in order to achieve an approximation to the current situation of the health network in the region. A reality that demands semantic changes to achieve more inclusive and participatory clinical and social responses in relation to mental health.

Keywords: Public Health; Mental Health; Health Systems; Social Support.

\section{Introducción}

El presente artículo analiza los resultados preliminares de una investigación vinculada a la Maestría en Salud Mental de la Universidad Nacional de Córdoba, que se enmarca en la Región Sanitaria I de la provincia de San Luis, Argentina. Se trata de la zona norte de la provincia, la cual está conformada por cuatro hospitales generales de los que dependen centros de salud. Las instituciones hospitalarias que participan de esta investigar son: Hospital de San Francisco, Hospital de Quines, Hospital de Luján y Hospital de Candelaria. La investigación parte de una aproximación a la realidad actual de los servicios de salud mental del interior, identificando sus potencialidades y necesidades desde la perspectiva de los profesionales que conforman los equipos de salud, gestores en salud y usuarios de los servicios. Asimismo, forma parte de este estudio la perspectiva de otros actores implicados en la salud mental y que se encuentran por fuera del sector salud, estos son: justicia, clubes deportivos, vivienda, municipio, escuelas, organizaciones civiles, entre otros. Todo ésto, con la meta de formular una propuesta de la reorganización en base a los postulados propuestos por el modelo de atención comunitaria en salud mental. Se adhiere en esta investigación a la definición de la salud mental desde un enfoque de salud integral, como un proceso determinado por componentes históricos, socio-económicos, culturales, biológicos y psicológicos, cuya preservación y mejoramiento implica una dinámica de construcción social vinculada a la concreción de los derechos humanos y sociales de toda persona ${ }^{1}$. Como modelo comunitario en salud mental, se entiende como condición básica para definir "lo comunitario" -el modelo, la práctica, la clínica- el respeto y garantía de derechos humanos. Las características principales de este modelo son: democrático, universal, equitativo, promocional, interdisciplinario, intersectorial, igualitario, accesible, presume capacidad, produce autonomía e inclusión social ${ }^{2}$. 
En relación a la atención de la salud mental en la provincia de San Luis, la experiencia de desinstucionalización, reconocida a nivel mundial, ha sido pionera en cuanto a procesos de reforma psiquiátrica y sanitaria. En el año 1993 se inició un proceso de transformación institucional que resultó en el cierre del hospital psiquiárico y su transformación en el actual Hospital Escuela de Salud Mental, un hospital de agudos. Este proceso culminó con la promulgación de la Ley de Desinstitucionalización (Ley № I- 0536-2006) que erradicó la institución del manicomio y el encierro como dispositivo de atención de la salud mental en la provincia, siendo los Derechos Humanos el eje rector del proceso, ${ }^{3,4}$.

En cuanto a la implementación de dispositivos sustitutivos al hospital psiquiátrico, se modificaron aspectos de éste tales como: internación de pacientes con la condición de que sea con su familia como acompañante, creación de diversos grupos como dispositivo fundamental de atención de patologías pisquiátricas, capacitación y formación de recursos humanos, servicio de domicilio, y se puso en marcha un arduo trabajo interinstitucional e intersectorial que requirió reuniones periódicas con sectores de justicia, educación, vivienda, trabajo, así como la creación e implementación del programa de Familias Sustitutas subsidiadas para acompañar a las personas que requerían externación y no contaban con ningún familiar ${ }^{5}$.

No obstante, a pesar de todos los avances tanto en la práctica como en materia legal y política en relación a la atención de la salud mental en la provincia de San Luis, actualmente dicha atención continúa siendo mayormente de manera centralizada, ubicándose el hospital monovalente en salud mental como principal y a veces único referente provincial. ¿Cambiar la modalidad del tratamiento sería suficiente? ¿Mantener un único espacio posible para el cuidado a las personas con problemas de salud mental no sería mantener en parte el modelo manicomial hospitalocéntrico? La falta de dispositivos comunitarios y de trabajo en red en el interior de la provincia genera grandes dificultades en el acceso a la atención y al mantenimiento de los tratamientos.

La fragmentación de los servicios de salud se manifiesta de múltiples formas en los distintos niveles del sistema de salud. En el desempeño general del sistema, como falta de coordinación entre los distintos niveles y sitios de atención, duplicación de los servicios y la infraestructura. En la experiencia de las personas que utilizan el sistema, la fragmentación se expresa como falta de acceso a los servicios, pérdida de la continuidad de la atención y falta de congruencia de los servicios con las necesidades de los usuarios ${ }^{6}$. Por todo esto, se consideró importante conocer las potencialidades $y$ fragilidades de los servicios de atención en salud mental y los dispositivos de atención comunitaria existentes en el interior de la provincia de San Luis, caracterizando la situación actual de la salud mental para pensar las vías posibles de reorganización de acuerdo al paradigma en salud mental comunitaria. 
En este sentido, han surgido interrogantes tales como ¿La fragmentación y la centralización de los servicios de salud generan dificultades en el acceso o mantenimiento de los tratamientos de los usuarios? ¿La atención de la salud de la comunidad requiere de intersectorialidad? ¿Es posible un trabajo en red con otros actores y sectores de la comunidad tales como justicia, deporte, vivienda, trabajo? ¿Será posible diversificar la formación de los recursos humanos en salud en pos de implementar dispositivos comunitarios de atención?

\section{Metodología}

La estrategia que se utiliza en el estudio implica considerar al mismo como una investigación cualitativa, la cual proporciona profundidad a los datos, dispersión, riqueza, flexibilidad, interpretativa, contextualización del ambiente o entorno, detalles y experiencias únicas ${ }^{7}$. Se trata estudio descriptivo en tanto intenta caracterizar y describir la situación actual de los servicios de salud de una región sanitaria teniendo en cuenta las necesidades y recursos de cada servicio con el objetivo de lograr una aproximación a la situación actual de los servicios de salud de la región. Teniendo en cuenta el tipo u origen de los datos, el estudio es un diseño de campo, ya que se obtiene directamente de la experiencia empírica, y se analiza datos primarios ${ }^{8}$. Dentro de éstos, se realiza un estudio de caso instrumental, es decir que no sólo se intenta conocer o comprender el caso sino que el interés se centra en un problema empírico o conceptual más amplio ${ }^{9}$.
La investigación se realiza en la región sanitaria I, zona norte de la provincia de San Luis, en las instituciones -servicios de salud y otras instituciones de la región como: escuelas, clubes deportivos, municipalidad, organizaciones civiles, comedores barriales, instituciones religiosas centros de jubilados, etc.- y en contacto con trabajadores, gestores, usuarios y otros actores sociales. Las Investigación fue aprobada por el Comité de Ética del Colegio de Psicólogos de la Provincia de San Luis.

La red de atención y las semánticas formales del cuidado

Para reflexionar sobre tales cuestiones, será importante conocer como está estructurado el sistema de salud argentino. Conocer también la atención en salud mental en la región estudiada, bajo a qué conceptos se organiza y sus semánticas de cuidado. Se parte de un sistema de salud en Argentina que está compuesto por tres subsectores: el Público, el de Obras Sociales y el Privado. En el sector público, el dispositivo que actualmente se presenta como oferta de servicios en el campo de la salud mental en la provincia de San Luis es el hospital. En el año 2000 se crea el Glosario de Términos Comunes en los servicios de salud del Mercosur, el cual define conceptos e incluso formas de actuación y organización de los servicios. Hospital: establecimiento de salud destinado a prestar asistencia sanitaria en régimen de internación a la población pudiendo disponer de asistencia ambulatoria u otros 
servicios. Los hospitales se dividen en Hospital General: hospital polivalente destinado a prestar asistencia a la salud en diferentes especialidades (clínica médica, salud mental cirugía, etc.); Hospital Especializado: hospital monovalente destinado a prestar asistencia a la salud en una especialidad (por ejemplo hospitales psiquiátricos). Otra definición compartida en este glosario, a su vez importante para estructurar una respuesta a los desafíos y potencialidades de la salud mental en esta provincia, es sobre las Redes de establecimientos de salud: conjunto de establecimientos del sistema de salud, regionalizado y jerarquizado por niveles de complejidad, capacitado para resolver todos los problemas de salud de la población de su responsabilidad. $Y$ sobre la forma en que actualmente está estructurada la atención en salud mental en la región, es necesario pensar sobre la derivación de los cuidados al hospital como la herramienta formal hegemónica. Una de las palabras más mencionadas en este tipo de organización de cuidado es Referencia: acto formal de envío de un paciente atendido en un determinado establecimiento de salud para otro de mayor complejidad; referencia siempre debería ser hecha después de constatarse la insuficiencia de la capacidad resolutiva, según normas y mecanismos preestablecidos ${ }^{6}$. Se entiende por servicios de salud en un sentido no restrictivo, a toda propuesta o alternativa de abordaje tendiente a la promoción de la salud mental, prevención del padecimiento, intervención temprana, tratamiento, rehabilitación, y/o inclusión social, reducción de daños evitables o cualquier otro objetivo de apoyo o acompañamiento que se desarrolle en los ámbitos públicos o privado ${ }^{10}$. Sin embargo, ¿que otras semánticas podemos accesar para realmente construir una red de atención en salud mental? Es cierto que la formalización de dispositivos y atributos de esta red ya está preestablecida en directrices y principios por los cuales están pautadas leyes y protocolos nacionales e internacionales sobre el tema. Principios y directrices consecuentes de la experiencia en otros territorios, incluso en otros países.

En este sentido, la OMS (2003) plantea que los servicios de salud deben tener los siguientes atributos: Accesibilidad: deben estar al alcance de toda la población tanto geográfica como cultural y administrativamente; Integralidad: disponer de recursos suficientes para satisfacer las necesidades de las personas a su cuidado; Coordinación y continuación de cuidados: que evite la fragmentación de la atención en el sistema de salud; Terrotorialidad o sectorización: que permita que un mismo equipo atienda a la población de toda el área asignada; Efectividad: prácticas basadas en técnicas validadas; Equidad: lo que determina el acceso a los servicios son las necesidades de las personas, y Respeto por los derechos humanos ${ }^{11}$.

En relación a los servicios de salud, se recomienda que sea un mismo equipo el que intervenga en las acciones de prevención y promoción, intervención terapéuticas y rehabilitación. Ésto se dificulta cuando se 
fragmentan los servicios por niveles de atención. En estos casos puede surgir como alternativa la constitución de pequeños equipos por barrios o asentamientos ${ }^{12}$.

Asimismo, en cuanto a los equipos de salud, se destaca la necesidad de la interdisciplina, comprendiendo este como un posicionamiento que obliga básicamente a reconocer la incompletud de las herramientas de cada disciplina. Incluye intercambios disciplinarios que producen enriquecimiento mutuo y transformación. Estos intercambios disciplinarios implican además interacción, cooperación y circularidad entre las distintas disciplinas, de manera sostenida y constante ${ }^{13}$.

\section{Semánticas de la salud mental comunitaria}

Levav Itzhack $^{14}$ nos enseña sobre la necesidad del desplazamiento de la atención de la salud mental desde el hospital psiquiátrico a la comunidad, planteando el siguiente esquema piramidal de distribución de funciones: 1) autocuidado; 2) grupos de autoayuda; 3) líderes comunitarios; 4) promotores de salud; 5) enfermeras; 6) médicos generales; 7) otros servicios de salud y 8) servicios especializados.

El modelo comunitario ${ }^{15}$ de atención propone que para lograr una mejora importante de la salud mental, es crucial que las personas reciban atención en sus propias comunidades. Ésto, permite que las mismas mantengan su integración social, conserven sus redes de apoyo social y sigan siendo miembros activos en la vida comunitaria. Es por eso que la atención comunitaria representa una manera eficaz, sostentible y humanitaria de mejorar la salud y el bienestar general.

Los servicios de salud mental deben abordar las necesidades de la población y ser sensibles al contexto. La organización de servicios de salud mental desde un modelo comunitario, corresponde a una red de servicios de salud mental sustitutiva al hospital psiquiátrico, como dice el propio nombre, con base comunitaria, acorde al proceso de desinstitucionalización. Este modelo se acoge al principio de integrar servicios de salud mental en la atención general de la salud y en otros servicios externos al ámbito de la salud, como son: educación, vivienda, empleo, justicia y bienestar social ${ }^{16}$.

\section{Semánticas del cambio semántico}

Pero la cuestión es más compleja. No basta adherir a las semánticas sanitarias del cuidado en salud mental. Belloc ${ }^{17}$ demuestra que una red de atención con muchos servicios, con una importante articulación intersectorial como en Cataluña (España), puede producir estigmatización, colonización de la experiencia de padecimiento y una suerte de institucionalización fuera de las gruesas paredes del manicomio. Una institucionalización a cielo abierto, cuando la vida de la persona está reglada por su diagnóstico, cuando su vida en todos los ámbitos está mediada por la definición biomédica de su padecimiento, cuando su vida 
es medicalizada y terapeutizada. En este sentido, apunta para la importancia del cambio de perspectiva en relación a quienes son las personas capaces de asistir en salud mental. Resulta fundamental que la locura deje de pertenecer a los profesionales, al campo psi, y restituir las determinaciones de la salud mental al campo de las prácticas y saberes cotidianos ${ }^{18}$. Un cambio semántico qué pasa por la reapropiación del protagonismo y de la contención comunitaria y cultural de la experiencia del padecimiento en salud mental. De la semántica de los especialistas, a la semántica del conocimiento que surge del modelo explicativo de la experiencia de padecimiento y sus posibilidades de articulación a acciones comunitaria e incluso a las prácticas y saberes cotidianos.

En relación a ésto, por medio de la imagen del "Iceberg de la Salud Mental" propuesta por Ferro $^{18,}$ se estima que un $80 \%$ de la población se encuentra con sufrimiento psíquico sin clasificación y sin asistencia por servicios oficiales de salud ya que son resueltos por la propia comunidad o por el autocuidado. Esto significa que la mayor parte de los problemas de salud mental se encuentra invisibilizada por las políticas públicas, pues el mismo autor demuestra que casi la totalidad de las inversiones en este campo están en los servicios especializados. Lo que también nos lleva a pensar que los $80 \%$ del iceberg de la salud mental, sumergidos de las políticas de salud mental, sólo salen a flote si tienen su experiencia de padecimiento interpretada como un cuadro psicopatológico, que en nuestra sociedad occidental conlleva la producción de estigma y una posible carrera sin vuelta de paciente psiquiátrico. ¿Hasta que punto no estamos produciendo iatrogenia, cronificación e institucionalización -aunque a cielo abierto-cuando para tener acceso a salud mental la persona necesita vincularse a un servicio especializado a si ya su experiencia de padecimiento? Esto sin hablar de los costes. Si pensamos en términos de salud general, al utilizar la alta complejidad de la atención para tratar de algo que pudiera ser resuelto en la atención primaria o en el nivel comunitario, sería como tratar una gripe común en el unidades de tratamiento intensivo.

Teniendo en cuenta la Pirámide Marco de Servicios de Salud Mental propuesta por la OMS en el año 2005, en la base de los servicios de salud mental nos encontramos con el autocuidado de la salud mental como capacidad de gobernar los intereses materiales, las relaciones sociales y los saberes de la vida cotidiana. Significa, por ejemplo, que las personas puedan detectar situaciones de alto riesgo para su salud mental y evitarlas, y que puedan adquirir aptitudes para enfrentar los problemas de la vida diaria ${ }^{19}$.

Sin embargo, se hace necesario una propuesta de semántica sanitaria donde otras semánticas y sus cambios y posibles giros dialécticos sea posible. En este sentido, encontramos una propuesta posible y potente en as redes de atención a la salud. Además, se plantea que el 
movimiento universal en busca de la construcción de redes de atención a la salud está sustentado por evidencias de que esas redes constituyen una forma estratégica de afrontar la crisis contemporánea de los sistemas de atención de salud, considerándose la implantación de las redes de atención de salud como una nueva forma de organizar el sistema. Se trata de implementar sistemas integrados que permitan responder, con efectividad, eficiencia, seguridad, calidad y equidad a las condiciones de salud de la población, promoviendo la participación y articulación comunitaria $^{17}$, produciendo, en el caso de las redes de atención a la salud mental, un necesario cambio semántico.

No existe un concepto unívoco de red, pero en general se usa el término para designar situaciones donde se visualizan intercambios no institucionalizados. El trabajo en red es una estrategia vinculatoria, de articulación e intercambio entre instituciones y/o personas que deciden asociar voluntaria o concertadamente sus esfuerzos, experiencias y conocimientos para el logro de fines comunes. La esencia del trabajo en red es la decisión de una o más personas, instituciones o áreas institucionales, de desarrollar una tarea en común, en procura de objetivos compartidos explícitos, manteniendo la identidad de los participantes. La intención del trabajo en red es aunar esfuerzos, evitar duplicaciones, alcanzar por complementariedad una mayor capacidad resolutiva, ser más eficaces y eficientes en lo que se hace $y$, todo ello como producto del intercambio y la colaboración. En tanto la perspectiva de red propone la concepción de un mundo construído por todos, nos coloca en situaciones de responsabilidades compartidas. Las características principales de las redes son: sistema abierto, lo cual implica que admite el ingreso y egreso de sus integrantes así como cambios en las funciones que desempeñan. Otro rasgo distintivo es la multicentralidad de la red, donde todo debe converger a y partir de un centro único de decisión. Otra característica importante de las redes de atención a la salud es la reciprocidad ${ }^{18}$.

La configuración red social implica un proceso de transformación permanente singular y colectivo, que acontece en múltiples espacios y asincrónicamente. Se puede imaginar como un sistema abierto, multicéntrico y heterárquico, que a través de la interacción permanente entre los actores de un colectivo (familia, equipo de trabajo, barrio, hospital, la escuela, asociación de profesionales, centro comunitario, entre otros) y con integrantes de otros colectivos, posibilita la potencialización de los recursos que poseen y la creación de alternativas novedosas para fortalecer la trama de la vida ${ }^{19}$.

La salud mental como semántica de/en transformación

Históricamente hemos visto muchos cambios en las prácticas en el campo de la salud mental, acompañadas por las luchas por derechos humanos y democratización del cuidado. Las reformas sanitarias y psiquiátricas por el mundo 
son ejemplos. No ha sido un progreso lineal, como les gustaría a los positivistas, sino que una compleja construcción con avances y retrocesos, una inmensidad de desafíos que se reorganizan y se actualizan, pero de grandes conquistas y potencialidades en el campo del cuidado en libertad, producción de vida e ciudadanía. En este sentido, una salud mental a partir del eje de la ciudadanía, por definición una construcción continua, una acción pautada por la libertad y la pluralidad ${ }^{20}$, tendrá necesariamente que operar desde una semántica de la transformación. Una semántica que se establece dialéticamente en su propia historia de transformaciones conceptuales, de la concepción de salud mental de un modelo asilar/manicomial a un modelo comunitario de atención así como experiencias de reforma de servicios sanitarios de atención de salud mental.

Durante la década de los 90, luego de la "Declaración de Caracas", se ha venido produciendo un expresivo cambio del modelo de atención en salud mental en Latinoamérica y en el Caribe. Se han impulsado políticas nacionales de salud mental, la reestructuración de los servicios psiquiátricos y la integración de lo psicosocial en la atención primaria en salud. El hospital psiquiátrico deja de ser el eje de la atención en salud mental, y pasa a ser brindada predominantemente por servicios de nivel secundario de atención y/o de base comunitaria 21 "La Declaración de Caracas" marcó un hito al plantear dos grandes objetivos: la superación del modelo de servicios psiquiátricos y la lucha contra todos los abusos y la discriminación de que son víctimas las personas con problemas de salud mental.

En el año 2001, la OMS/OPS insta a los Estados a incluir la salud mental entre las prioridades de la salud pública, reforzar el enfoque multisectorial de la salud mental y promover la participación familiar y comunitaria y fundamentalmente continuar realizando esfuerzos para la reorientación de los servicios de salud mental (de institucionales a comunitarios). En el 2005, se celebró en la capital del Brasil, la "Conferencia Regional para la Reforma de los Servicios de Salud Mental: 15 años después de Caracas", en la que se focalizó especialmente en la reforma de los servicios y en la protección de los derechos humanos de las personas con trastornos mentales.

La OMS en el Plan de Acción sobre Salud Mental (2013), recomienda el desarrollo de servicios integrales de salud mental y de asistencia social de base comunitaria; la integración de la asistencia y los tratamientos de salud mental en los hospitales generales y la atención primaria y la continuidad asistencial entre diferentes prestadores y niveles del sistema de salud. América Latina y el Caribe han demostrado que es posible mejorar la atención en salud mental en los países de la región cuando existe voluntad política, asignación de recursos de acuerdo a la importancia relativa de la salud mental y capacidad técnica para implementar las reformas necesarias ${ }^{21-23}$.

Respecto a las experiencias de reformas en la atención de la salud mental en todo el mundo 
ha sido variada, respondiendo a factores políticos, económicos, sociales, culturales, de cada país y región geográfica que han ido produciendo sus propios procesos de construcción de políticas públicas en relación a la salud y a la salud mental en particular.

Para el caso de Argentina, la Dirección Nacional de Salud Mental y Adicciones, la Ley Nacional de Salud Mental № 26657(2010) con su decreto reglamentario (2013), así como el Plan Nacional de Salud Mental (2013), son instrumentos concretos que permiten avizorar cambios profundos en el sistema de salud mental argentino. Se entiende, a partir de esto, que la Salud Mental aspira al desarrollo de acciones territoriales al alcance de toda la población desde la mirada comunitaria y la inclusión social. Se enfatiza en la promoción de procesos de atención basados en abordajes interdisciplinarios e intersectoriales, integrados por profesionales, técnicos y otros trabajadores capacitados para la atención en salud mental, por fuera del ámbito de la internación hospitalaria monovalente (manicomios), basado en los principios de la Atención Primaria en Salud (APS), orientándose al reforzamiento, restitución y promoción de los lazos sociales ${ }^{23}$.

La situación de cada provincia en el país en relación a su política y organización de servicios de salud es diferente. Se destacan las experiencias pioneras de Rio Negro del año 1985 y de San Luis en 1993 anteriormente descripta. En relación a la primera en el año 1991, se inició una profunda transformación en salud mental, de "desmanicomialización", en la que se cerró el hospital psiquiátrico y se sancionó la Ley Provincial № 2440 "Promoción Sanitaria y Social de las Personas que Padecen Sufrimiento Mental". En ésta se establece la apertura de dispositivos de atención con base territorial y de servicios de Salud Mental en los hospitales generales de esa provincia, la conformación de equipos interdisciplinarios para atender problemáticas mentales en sus lugares de origen, y un intenso trabajo comunitario con el eje puesto en la inclusión social de las personas con padecimiento mental. Dicha experiencia de desmanicomialización fue un proceso colectivo, progresivo y participativo en el que se incluyó a los usuarios, familias y comunidad como actores claves en el proceso de transformación ${ }^{24-25}$.

Por último, se destaca la diversidad de situaciones actuales respecto al cumplimiento de la Ley Nacional de Salud Mental en las provincias, así como la existencia de leyes provinciales de salud mental, las cuales están en vigencia en las provincias de: Santa Fe, Salta Entre Ríos, San Juan, Santa Cruz, Córdoba, Buenos Aires, Chubut y San Luis ("Ley de desinstitucionalización").

Sin embargo, hay un dicho popular triestino que dice "la carta no canta". Tal vez no sea una simple coincidencia que en Trieste (Italia), donde se ha producido una de las más conocidas experiencias de reforma psiquiátrica y procesos de desinstitucionalización, se sabe 
en el sentido común que quien canta son las personas y no la letra fría. Es decir que si no encarnamos esa letra, si no transportamos para nuestro gesto cotidiano de gestión y atención en salud mental las conquistas colectivas que se manifiestan en las leyes, nunca habrá construcción ciudadana y de derechos humanos. La carta canta si sus letras están vivas en el trabajo, en el cuidado construido a la par con el usuario. En este sentido, la Ley Nacional de Salud Mental de Argentina, así como las leyes provinciales, sólo podrán ser realmente implementadas en la medida en que estén vivas en el cotidiano de actos de salud de trabajadores, gestores yusuarios.

\section{Semánticas de la transformación en la salud mental de San Luis}

Teniendo como eje esas acciones vivas en el cotidiano de trabajadores, gestores, usuarios, de una semántica de la transformación que pueda derrumbar las paredes de los manicomios mentales ${ }^{31}$-que se establece desde la micropolítica hasta la macropolítica-, que pueda protegernos de nuestros propios crímenes de la paz ${ }^{32}$, buscamos formular una propuesta de reorganización en los servicios de atención de salud mental en la Región Sanitaria 1 del interior de la provincia de San Luis desde un modelo de atención comunitaria en salud. En el itinerario de esta formulación necesitamos identificar los recursos y necesidades de los servicios de salud mental de la región, describir la situación actual de los servicios de salud mental, así como desarrollar lineamientos para una propuesta de reorganización de tales servicios y su articulación otro sectores de otras políticas públicas, movimientos sociales y organizaciones de la comunidad, es decir, en un modelo de atención comunitaria en salud.

Y de las semánticas que alejan las personas diagnosticadas por problemas de salud mental, desde la ubicación de su proceso de salud/enfermedad/atención ${ }^{33}$ en servicios especializados en salud mental, hasta la propia iatrogenia vinculada a una vida colonizada por el rol de diagnosticado, el reto no es otro que la creación de una poética ciudadana. Se trata de construir colectivamente una red que pueda apoyar esta experiencia de padecimiento, tratarla comunitariamente, sin romantizar un proceso que produce innegable sufrimiento. Consolidar condiciones comunitarias pasa por agregar a la red instituciones, grupos, movimientos sociales, o incluso ciudadanos, sin vinculación directa al sector salud, especialmente sin la marca de la especialidad de salud mental. La idea es una salud mental no apartada de la vida normal y corriente. Como un poema de una gran obra, se trata de una red construida por los versos singulares de cada uno de los participantes, que se inscriben y deialéticamente construyen un modelo de sociedad más inclusivo y plural. 


\section{Referencias}

1. Argentina. Boletín Oficial de la República Argentina, N³2041. Ley Nacional de Salud Mental. Ley 26657/2010. Argentina; 2010.

2. Stolkiner A. Interdisciplinar y Salud Mental. Ponencia presentada en IX Jornadas Nacionales de Salud Mental y I Jornadas Provinciales de Psicología. Salud Mental y Mundialización: Estrategias posibles en la Argentina de hoy. Posadas, Misiones, Argentina; 2005.

3. Pellegrini, J. Salud es sin encierros. San Luis: Editorial Payné; 2009.

4. Senado y Cámara de Diputados de la Provincia de San Luis. Ley de Desinstitucionalización (Ley № I- 0536-2006). San Luis; 2006.

5. Pellegrini J. Cuando el manicomio ya no está. San Luis: Editorial Payné; 2011.

6. Organización Panamericana de la Salud. La renovación de la atención primaria de salud en las Américas: documento de posición de la OPS/OMS. Washington, D.C; 2007.

7. Hernández Sampieri R, Fernández Collado C. Metodología de la investigación. 6a ed. México: McGrawhill/Interamericana; 2014.

8. Sabino C. El Proceso De Investigación [Internet]. Caracas: Panapo; 1992.

9. Stake RE. Investigación con estudio de casos. Morata, 4ae ed, Madrid: Morata; 2007.

10. Buenos Aires. Gobierno de la Ciudad Autónoma de Buenos Aires (GABA). Glosario Servicios de Salud del Mercosur. MERCOSUR/GMC/RES N²1/00; 2000. Recuperado en: http://www.bvs.org.ar/pdf/serviciosdesalud.pdf

11. Argentina. Boletín Oficial de la República Argentina, $n^{\circ}$ 45737. Decreto Reglamentario Ley Nacional de Salud Mental 603/2013. Argentina; 2013.

12. World Health Organization. Mental health policy and service guidance package, Organization of Services for Mental Health, Geneva; 2003.

13. Cohen, H. De la Desinstitucionalización a la Atención en la Comunidad. Capítulo 9. En: Manual de Salud Mental en la comunidad. 2da edición. Washington, D.C; Editorial: PALTEX, 2009.

14. Levav I. Temas de salud mental en la comunidad. Organización Panamericana de la Salud. Washington: Paltex; 1992.

15. Natella G. Salud Mental Comunitaria en acción: el problema de la formación profesional. En: Salud Mental, Comunidad y Derechos Humanos. Universidad de la Republica del Uruguay: Psicolibros Universitaria; 2017.

16. Funk M, Faydi E et al. Organización de los servicios de salud mental. Capítulo 8. El modelo comunitario en salud. En: Salud Mental en la Comunidad. 2a ed. Washington: Paltex; 2009.

17. Belloc MM. Homem-sem-história. A narrativa como criação de cidadania [tese]. Universitat Rovira i Virgili. Tarragona; 2011.

18. Ferro R. Salud Mental y Poder. Un abordaje estratégico de las acciones en salud mental en la comunidad. Revista Escuela de Salud Pública. 2010 14(2):47-62.

19. Ferro R. Formación de recursos humanos en salud mental en la comunidad. Capítulo 15. En: Salud Mental en la Comunidad. 2da edición. Washington, D.C; Editorial: PALTEX; 2009.

20. Mendes E. Las redes de atención de salud. Organización Panamericana de la Salud. Brasil, D-F; 2011.

21. Ministério de la Salud. Argentina. Manual de Posgrado en salud social comunitaria. Programa de médicos c omunitarios. Módulo 9: Salud y Redes. Disponible e n : http://www.msal.gob.ar/images/stories/bes/graficos/0000001033cnt-modulo_8_trabajo-equipos-salud.pdf

22. Dabas E: ¿Quién sostiene a las familias que tienen que sostener a los niños? Redes sociales y restitución comunitaria. Revista terapia y Familia. Vol 16. N⒉ México; 2003.

23. Arendt H. La condición humana. 1ạ ed. Buenos Aires. Paidos; 2009.

24. Rodríguez J. Protección de la salud mental en situaciones de desastres y emergencias. Capítulo 20. In: Salud Mental en la Comunidad. 2da edición. Washington, D.C; Editorial: PALTEX; 2009.

25. Organización Mundial de la Salud. Plan de Acción sobre Salud Mental (2013-2020). Ginebra, Suiza; 2013.

26. Organización Mundial de la Salud. Organización Panamericana de la Salud. La reforma de los servicios de salud mental: 15 años después de la Declaración de Caracas; Washington, D.C; 2007. 
27. Dimenstein M. La reforma psiquiátrica y el modelo de atención psicosocial en Brasil: en busca de cuidados continuados e integrados en salud mental. Revista CS 201343-71. Disponible en: http://www.redalyc.org/articulo.oa?id=476348374002.

28. Argentina. Plan Nacional de Salud Mental 2013-2018. Resolución 2177/2013. Ministerio de Salud, República Argentina; 2010.

29. Río Negro. Boletín oficial de la Provincia de Rio Negro № 2909. Ley № 2440, Promoción Sanitaria y Social de las Personas que Padecen Sufrimiento Mental", Rio Negro; 1991.

30. Cohen H, Natella G. Argentina: El Programa de salud mental en la provincia de Río Negro. Capítulo 28. En: Salud Mental en la Comunidad. 2da edición. Washington, D.C; Editorial: PALTEX; 2009.

31. Pelbart PP. Manicômio mental: a outra face da clausura. In: Lancetti A, organizador. Saúde e loucura nㅇ 2.3 ed. São Paulo: Hucitec; 1990. p.131-38.

32. Basaglia F, Ongaro FB, organizadores. Los crímenes de la paz: investigación sobre los intelectuales y los técnicos como servidores de la opresión. México: Siglo Veinte Uno Editores; 1987.

33. Menéndez EL. Sujeitos, saberes e estruturas: uma introdução ao enfoque relacional no estudo da saúde coletiva. São Paulo: Hucitec; 2009.

Submissão: $18 / 02 / 2020$

Aceite: 19/04/2020 